\title{
Indoor Localization Based on Time of Arrival Using Multi-Zone Technique
}

\author{
Mahmood F. Mosleh ${ }^{1}$, Alhamzah T. Mohammad ${ }^{2}$, Ahmed k. Daraj $^{3}$ \\ $\left\{\right.$ drmahmood@mtu.edu.iq ${ }^{1}$, alhamza_tm@yahoo.com², ahmed.daraj@gmail.com $\left.{ }^{3}\right\}$ \\ Middle Technique University, Baghdad, Iraq ${ }^{123}$
}

\begin{abstract}
Indoor position localization suffers from the regressive signal when the system implement based on Receive Signal Strength (RSS) and time synchronization when implement based on Time of Arrival (ToA). Those problems lead to high error in terms of position localization. To mitigate those errors many techniques and methods are deployed such as NLOS identification, Maximum Likelihood (ML), and weighted least-squares (WLS). In this paper, a new technique based on optimization of transmitter location has been proposed by separating the case study building into multi-zone, each zone having specific parameters which defer from other ones. The deployed receivers were 8 for the zones $(A-F)$ and 20 into the zone $G$ to be 68 for the whole system. There are three transmitters deployed with a changeable position to select a better one. The results confirm that the error improved from $(0.638 \mathrm{~m})$ based on individual TOA to $(0.136 \mathrm{~m})$ by using propose Multi-zone ToA, while improved from $(0.756 \mathrm{~m})$ rely on individual RSS to $(0.1223 \mathrm{~m})$ based on Multi-Zone RSS.
\end{abstract}

Keywords: ToA, RSS, indoor localization, multi-zone.

\section{Introduction}

Indoor localization gains attention and popular topic for researchers recently to locate the person and any other object. The indoor localization constructed to correspond with the crowded building to track the children, patient movements, monitoring, and security. The common technique widely used to find location is the Global Position System (GPS). However, GPS is convenient in outdoor environments and there are constraints and limitations that obscured GPS service like the position between high building or inside building that disconnect satellite with targets [1]. In this situation, indoor localization systems are used to estimate the position in any area that GPS doesn't work so well in it. For each environment, several methods are used such as, Time of Arrival (ToA) [2], [3], [4], Time Deference of Arrival (TDoA) [5], Angle of Arrival (AoA) [6], and Receive signal strength (RSS) [7], [8], [9], [10], [11]. Also, there are hybrid methods such as, ToA/AoA [12], RSS/ToA [13], [14], and TDoA/RSS [15]. In general, signal characteristics use to construct the indoor localization systems in most projects to estimate the distance between reference points (RPs) and targets. In addition, Wireless Fidelity (Wi-Fi) is proliferate everywhere, subsequently, a lot of indoor localization systems design based on WiFi [16], [17], [18], [19]. However, indoor wireless communications suffer from several 
constraints and challenges that impact system reliability. The phenomenon widely recognized effect on system accuracy called multipath which happens in Non-Line of Sight (NLoS) environments when obstacles separate transmitters and receivers [1]. Furthermore, time synchronization and time measurement are common problems in inferring location using ToA [20]. To address those problems, many researchers proposed several techniques and algorithms like [21], [22], whose proposed NLOS identification technique to alleviate the impact of multipath. Also, Maximum Likelihood (ML) and weighted least-squares (WLS) have been formulating for indoor localization [23], [24]. To improve position systems based on RSS, WiFi fingerprint also has already been proposed in [25], [26]. In this paper, the Multi-Zone technique will propose to mitigate the NLOS phenomenon and complexity into a crowded building. Such a propose technique aims to separate the case study building into seven zones each one having a specific parameter such as path loss exponent and noise value. In addition, to mitigate the regressive signals and find suitable coverage to whole targets, optimum position (OP) will determine to deploy the transmitters. Both procedures aim to carry out an optimum indoor localization system with high accuracy.

\section{Localization based ToA \& RSS}

The estimated distance between RPs and targets can be estimated depending on spent time needs to reach receivers, where RPs could be access points or anchor points while the targets is the receives points. The exact distance can be calculated as [2]:

$$
d_{i}=\left\|x-x_{i}\right\|=\sqrt{\left(\mathrm{x}-x_{i}\right)^{2}+\left(\mathrm{y}-y_{i}\right)^{2}} \quad i=1.2 \ldots . k
$$

The distance between RPs and targets can be calculated depending on TOA as following [27]:

$$
d_{i}=C *\left(\text { time }_{\text {arrival }}-\text { time }_{\text {sent }}\right)
$$

Where $\mathrm{C}$, is the speed of light $\approx 3 * 10^{8}(\mathrm{~m} / \mathrm{s})$. As mentioned above, there are constraints due to multipath environments. Therefore, (1) can be written as following [2]:

$$
d_{i}=\left\|x-x_{i}\right\|+\beta_{i}+m_{i}
$$

Where $\beta_{i}(m)$ equal to 0 in LOS and greater than 0 in NLoS, $m_{i}$ is noise value.

To estimate targets position, reference path loss calculates in one meter which recommended in [20]. In addition, the path loss for the distance between transmitters and receivers can express as $[2,20]$ :

$$
P L(d)=P l o-b_{i}-10 \gamma \log 10 \frac{\left\|x-x_{i}\right\|}{d_{o}}
$$


Where Plo IS reference path loss, $b_{i}$ is positive bias, $\gamma$ is path loss exponent, $z$ is the lognormal shadowing and can express as a zero-mean Gaussian random variable,

i.e. $n_{i} \sim N\left(0, \sigma_{n i}^{2}\right)$ and $m_{i} \sim N\left(0 . \sigma_{m i}^{2}\right)$ is the level of noise (m).

When measurements of ToA and RSS presented, then can do some manipulation by list the both RSS and TOA as one vector, $p=\left[p_{i}\right]^{T}$ and $d=\left[d_{i}\right]^{T},\left(p . d \in \mathbb{R}^{N}\right)$. Then joint likelihood function can write as following [23]:

$$
\begin{gathered}
\Lambda\left(p . d \mid x . b_{i} \cdot \beta_{i}\right)=p\left(p \mid x . b_{i}\right) p\left(d \mid x . \beta_{i}\right)=\frac{1}{\sqrt{2 \pi \sigma_{n i}^{2} \sigma_{m i}^{2}}} \\
\exp \left\{-\frac{\left(p i-p o+b i+10 \gamma \log _{10} \frac{\| x-x i||}{d 0}\right)^{2} \sigma_{m i+(d i|| x-x i \|-\beta i)^{2} \sigma_{n i}^{2}}^{2}}{\sigma_{n i}^{2} \sigma_{m i}^{2}}\right\}
\end{gathered}
$$

Also, the hybrid $M L$ for $\left(x \cdot b_{i} \cdot \beta_{i}\right)$. can be written as following [28]:

$$
\{x . b i . \beta i\}=\operatorname{argmin} \sum_{i=1}^{N} x . b i . \beta i
$$

However, according to [23], the equation above does not close from exact results also it's difficult to distinguish between LOS and NLoS. Accordingly, hybrid ML is not a perfect solution for multipath impact. The authors in [23] suggested an integrated ToA and RSS to employ the advantage for both techniques which can mitigate the corresponding drawback for each one, such estimate distance calculates depending on RSS and ToA as following:

$$
\begin{aligned}
& \left(d_{i}\right)^{R S S}=10^{\frac{p o-p i-\frac{b^{\wedge} \max }{2}}{10 \gamma}} \\
& \left(d_{i}\right)^{\text {TOA }}=d_{i}-\frac{\beta}{2}^{\max }
\end{aligned}
$$

The normalized of (7) and (8) can be calculate as following [23]:

$$
\in=\frac{\left|d_{i}^{R S S}-d_{i}^{T O A}\right|}{\max \left\{d_{i}^{R S S} \cdot d_{i}^{T O A}\right\}}
$$

To estimate targets located, trilateration method could be applied as shown in figure (1) which depending on distances between RPs and targets [1], [20]:

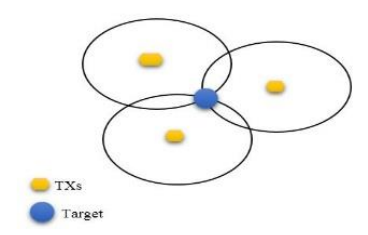

Fig.1. Estimate position using trilateration methods 


\section{Case study}

In this paper, the Wireless Insite Package is applied to design the selected case study building. The campus of electrical engineering technical college is the experimental case study. Such a building consists of three floors; our experiment is applied on the first floor only. Such a floor is separated into 7 zones (A-G) related to the content of each zone such as, walls, objects, and types of materials that impact propagation patterns to give specific parameters for each zone. The receiving point is distributed as 68 receivers 8 for each zone except zone $\mathrm{G}$ with 20 receivers. The number of transmitters is three with a changeable position to select a better one.

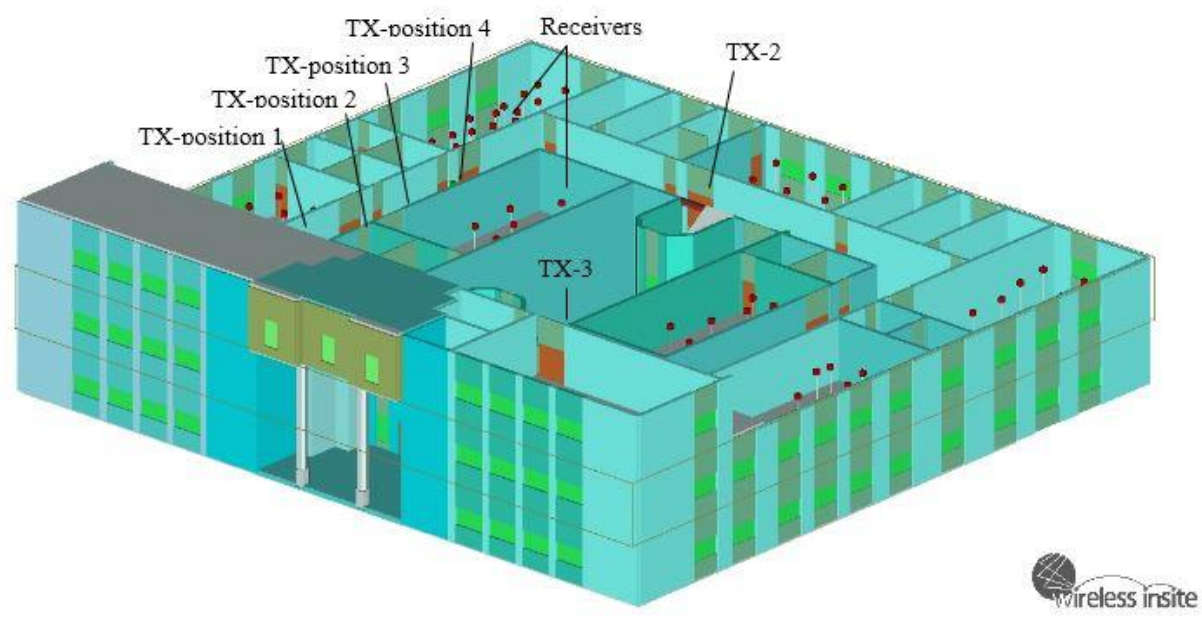

Fig. 2. Transmitters position that selected

In this research, $4.5 \mathrm{GHZ}$ frequency was selected in addition to an omnidirectional antenna for transmitters and receivers have been used as shown in Table (1).

Table 1: The characteristics of antennas

\begin{tabular}{ccc}
\hline Antenna properties & TX Antenna & RX Antenna \\
\hline Antenna type & Omni-Directional & Omni-Directional \\
Input Power $(\mathrm{dBm})$ & 15 & - \\
Gain $(\mathrm{dBi})$ & 7 & 1 \\
E-Plane HPBW & $10^{\circ}$ & $90^{\circ}$ \\
Waveform & Sinusoid & Sinusoid \\
Temperature $(\mathrm{k})$ & 293 & 293 \\
Polarization & $\mathrm{V}$ & $\mathrm{V}$ \\
Received Threshold $(\mathrm{dBm})$ & -130 & -130 \\
\hline
\end{tabular}




\section{Results \& Discussion}

The performance of indoor localization using ToA with the proposed technique, illustrated in figure (3a). In OTP with a multi-zone technique, the position of transmitter played the main role to improve the performance of systems by decrease distance error. To find OTP, the position of the transmitter has been changed 4 times. The average distance error in the fourth position is $(0.136 \mathrm{~m})$. However, the first position was the worst one with average distance error $(0.638 \mathrm{~m})$.

In addition, position estimation based on RSS method has been improved by using the proposed technique. As mention, the regressive RSS is the main challenge. By using OTP, the long-distance can be alleviated, subsequently, the performance of the system will be improved. Figure (3b) illustrates the distance error in each transmitter's position. The fourth position is better than others with average distance error $(0.1223 \mathrm{~m})$ even better than ToA. In contrast, the first position was the worst one with average distance error $(0.756 \mathrm{~m})$ and that illustrates the impact of the long-distance on RSS measurements to implement indoor localization. 


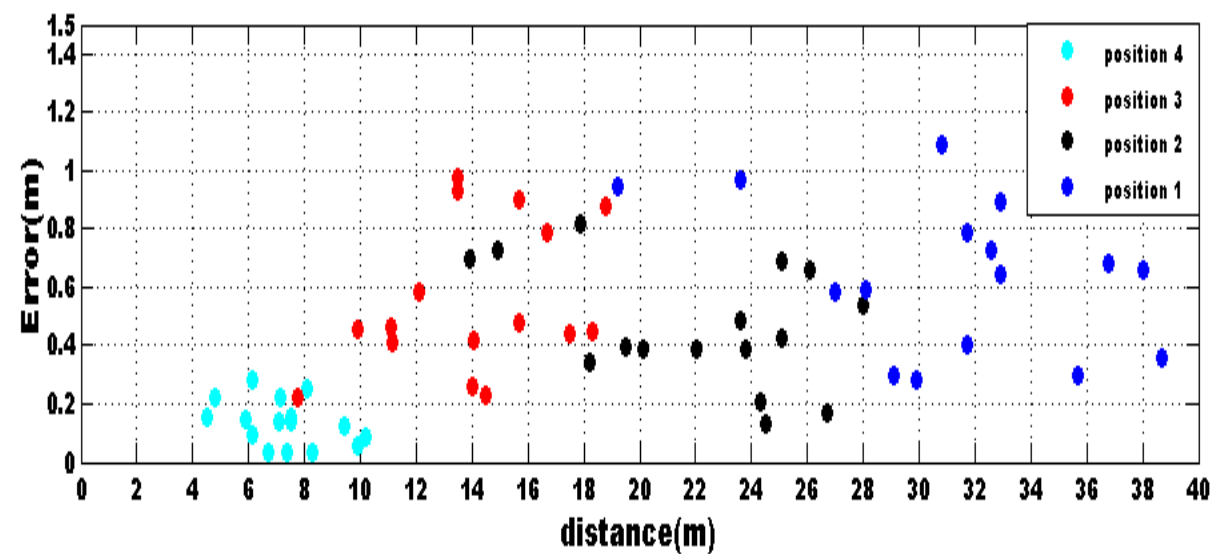

Fig.3a. Distance error in difference TX position using TOA method

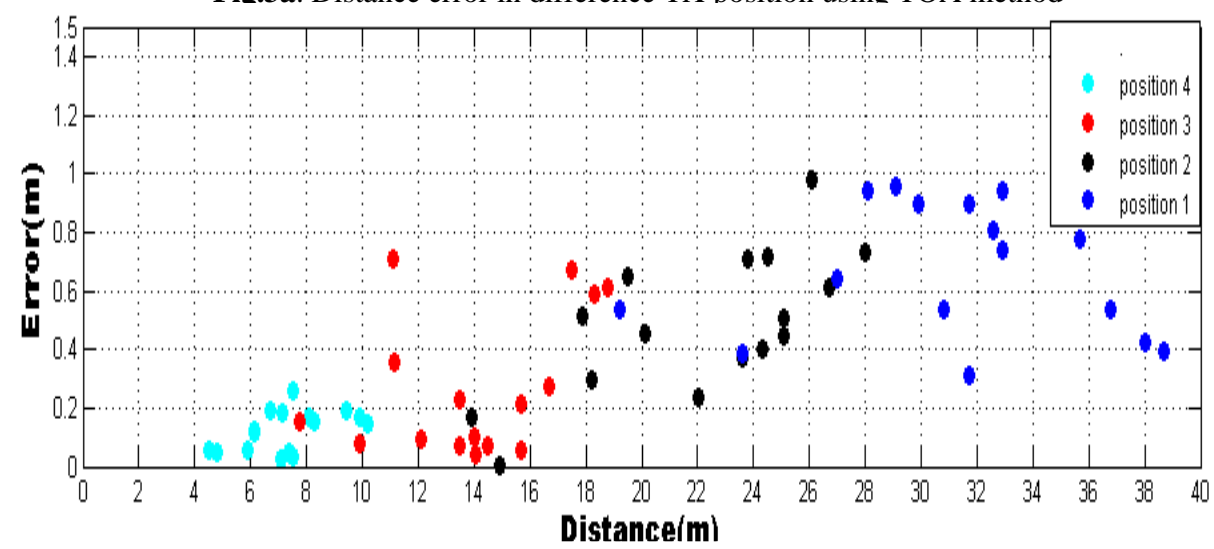

Fig.3b. Distance error in difference TX position using RSS method

\section{Conclusions}

In this research, a proposed OTP technique is applied with each RSS and ToA in order to find locations of 68 targets deployed in a preplanned manner in a selected building. Such a building has been simulated using the Wireless Insite package to apply the proposed techniques in order to show the performance of position locations. Three transmitters based on $4.5 \mathrm{GHz}$ are deployed in a selected place within a case study building. The results confirm that the error decreases significantly (which calculated as the differences between exact and estimated position) based on the proposed OTP combined with RSS and ToA. Also, the results show that such error has been enhanced from $(0.638 \mathrm{~m})$ to $(0.136 \mathrm{~m})$ using the proposed OTP. In contrast, the OTP with RSS evolves the errors from $(0.756 \mathrm{~m})$ to $(0.1223 \mathrm{~m})$ which confirms that OTP with RSS is slightly outperformed OTP with ToA. 


\section{References}

[1] Zekavat, Reza, and R. Michael Buehrer: Handbook of position location: Theory, practice and advances. 1'st ed., Vol. 27. pp.5-867 John Wiley \& Sons, (2011).

[2] Tomic S, Beko M, Dinis R, Montezuma P: A robust bisection-based estimator for TOA-based target localization in NLOS environments. IEEE Communications Letters. vol. 21, no. 11, pp. 2488-2491 (2017).

[3] Khalaf-Allah M: Time of arrival (TOA)-based direct location method. In 2015 16th International Radar Symposium (IRS). Jun 24 (pp. 812-815). IEEE (2015)

[4] Xu, Enyang, Zhi Ding, and Soura Dasgupta: Wireless source localization based on time of arrival measurement. In 2010 IEEE International Conference on Acoustics, Speech and Signal Processing, pp. 2842-2845. IEEE, (2010).

[5] Han Z, Leung CS, So HC, Constantinides AG: Augmented Lagrange programming neural network for localization using time-difference-of-arrival measurements. IEEE transactions on neural networks and learning systems.pp. 3879-84 (2017).

[6] Tomic S, Beko M, Dinis R: Distributed RSS-AoA based localization with unknown transmit powers. IEEE Wireless Communications Letters. pp. 3879-3884, (2016).

[7] Kejiong Li: Indoor and Outdoor Location Estimation in Large Areas Using Received Signal Strength. University of London. July, (2013).

[8] B. Jia, B. Huang, H. Gao, W. Li, and L. Hao: Selecting Critical WiFi APs for Indoor Localization Based on a Theoretical Error Analysis. IEEE Access, vol. 7, pp. 36312-36321, (2019).

[9] Y. Hu and G. Leus: Robust Differential Received Signal Strength-Based Localization. IEEE Trans. Signal Process., vol. 65, no. 12, pp. 3261-3276, (2017).

[10] Z. Kaibi, Z. Yangchuan, and W. Subo: Research of RSSI indoor ranging algorithm based on Gaussian - Kalman linear filtering. Proc. 2016 IEEE Adv. Inf. Manag. Commun. Electron. Autom. Control Conf. IMCEC 2016, no. 3, pp. 1628-1632, (2017).

[11] A. Achroufene, Y. Amirat, and A. Chibani: RSS-Based Indoor Localization Using Belief Function Theory. IEEE Trans. Autom. Sci. Eng., vol. 16, no. 3, pp. 1163-1180, (2019).

[12] Y. Y. Li, G. Q. Qi, and A. D. Sheng: Performance Metric on the Best Achievable Accuracy for Hybrid TOA/AOA Target Localization. IEEE Commun. Lett., vol. 22, no. 7, pp. 1474-1477, (2018).

[13] Catovic A, Sahinoglu Z: The Cramer-Rao bounds of hybrid TOA/RSS and TDOA/RSS location estimation schemes. IEEE Communications Letters. pp. 626-8 (2004).

[14] Tiwari S, Wang D, Fattouche M, Ghannouchi F: A hybrid RSS/TOA method for 3D positioning in an indoor environment. ISRN Signal Processing. (2012).

[15] S. Tiwari et al: Practical result of wireless indoor position estimation by using hybrid TDOA/RSS algorithm: Can. Conf. Electr. Comput. Eng., no. 1, pp. 1-5, (2010).

[16] C. Chen, Y. Chen, H. Q. Lai, Y. Han, and K. J. R. Liu: High accuracy indoor localization: A WiFibased approach. ICASSP, IEEE Int. Conf. Acoust. Speech Signal Process. - Proc., vol. 2016-May, pp. 6245-6249, (2016).

[17] S. A. Yuvaraj and L. C. Siddanna Gowd: High performance implementation of RSSI based Wi-Fi location tracker for android applications. J. Theor. Appl. Inf. Technol., vol. 71, no. 1, pp. 97-106, (2015). [18] X. He, S. Badiei, D. Aloi, and J. Li: WiFi iLocate: WiFi based indoor localization for smartphone. Wirel. Telecommun. Symp., (2014).

[19] B. Kim, W. Bong, and Y. C. Kim: Indoor localization for Wi-Fi devices by cross-monitoring AP and weighted triangulation. 2011 IEEE Consum. Commun. Netw. Conf. CCNC'2011, pp. 933-936, (2011)

[20] M. Werner: Indoor location-based services: Prerequisites and foundations, vol. 9783319106991. (2014).

[21] İ. Güvenç, C.-C. Chong, F. Watanabe, and H. Inamura: NLOS Identification and Weighted LeastSquares Localization for UWB Systems Using Multipath Channel Statistics. EURASIP J. Adv. Signal Process., vol. 2008, no. 1, (2007).

[22] S. Maranò, W. M. Gifford, H. Wymeersch, and M. Z. Win: NLOS identification and mitigation for localization based on UWB experimental data. IEEE J. Sel. Areas Commun., vol. 28, no. 7, pp. 10261035, (2010). 
[23] S. Tomic and M. Beko: Target localization via integrated and segregated ranging based on RSS and TOA measurements. Sensors (Switzerland), vol.. 19, no. 2, pp. 1-20, (2019).

[24] M. Pauley and J. H. Manton: The existence question for Maximum-Likelihood estimators in Timeof-Arrival-Based localization. IEEE Signal Process. Lett., vol. 25, no. 9, pp. 1354-1358, (2018).

[25] X. Wang, X. Wei, Y. Liu, K. Yang, and X. Du: Fingerprint-based Wi-Fi indoor localization using map and inertial sensors. Int. J. Distrib. Sens. Networks, vol. 13, no. 12, (2017).

[26] Mosleh MF, Abd-Alhameed RA, Qasim OA: Indoor Positioning Using Adaptive KNN Algorithm Based Fingerprint Technique. InInternational Conference on Broadband Communications, Networks and Systems, (pp. 13-21). Springer, Cham (2018).

[27] O'Keefe, Brian: Finding Location with Time of Arrival and Time Difference of Arrival Techniques. ECE Senior Capstone Project (2017).

[28] A. K. Kutai and B. Y. Gurevich,: Method of determining an abutting line or plane from measurement data. .Meas. Tech., vol. 11, no. 9, pp. 1268-1273, (1968). 